\title{
Simultaneous Aerobic Exercise and Memory Training Program in Older Adults with Subjective Memory Impairments
}

\author{
Sarah C. McEwen ${ }^{\mathrm{a}, \mathrm{b}, 1, *}$, Prabha Siddarth $^{\mathrm{b}, 1}$, Berna Abedelsater ${ }^{\mathrm{b}}$, Yena Kim ${ }^{\mathrm{c}}$, Wenli Mui ${ }^{\mathrm{b}}$, \\ Pauline $\mathrm{Wu}^{\mathrm{b}}$, Natacha D. Emerson ${ }^{\mathrm{b}}$, Jacob Lee ${ }^{\mathrm{b}}$, Shayna Greenberg ${ }^{\mathrm{b}}$, Tiffany Shelton ${ }^{\mathrm{b}}$, Scott Kaiser ${ }^{\mathrm{c}}$, \\ Gary W. Small ${ }^{\mathrm{b}}$ and David A. Merrill ${ }^{\mathrm{b}}$ \\ ${ }^{a}$ Department of Psychiatry, School of Medicine at UCSD, La Jolla, CA, USA \\ ${ }^{\mathrm{b}}$ Department of Psychiatry and Biobehavioral Sciences, David Geffen School of Medicine at UCLA, and Semel \\ Institute for Neuroscience and Human Behavior, Los Angeles, CA, USA \\ ${ }^{\mathrm{c}}$ Motion Picture Television Fund, Woodland Hills, CA, USA
}

Handling Associate Editor: Cyrus Raji

Accepted 19 December 2017

\begin{abstract}
.
Background: Several modifiable lifestyle factors have been shown to have potential beneficial effects in slowing cognitive decline. Two such factors that may affect cognitive performance and slow the progression of memory loss into dementia in older adults are cognitive training and physical activity. There are currently no effective treatments for dementia; therefore, preventative strategies to delay or prevent the onset of dementia are of critical importance.

Objective: The aim of this study was to determine the relative effectiveness of simultaneous performance of memory training and aerobic exercise to a sequential performance intervention on memory functioning in older adults.

Methods: 55 older adults (aged 60-75) with subjective memory impairments (non-demented and non-MCI) completed the intervention that consisted of 90-minute small group classes held twice weekly. Participants were randomized to either 4weeks of supervised strategy-based memory training done simultaneously while stationary cycling (SIM) or sequentially after the stationary cycling (SEQ). Standardized neurocognitive measures of memory, executive functioning, speed of processing, attention, and cognitive flexibility were assessed at baseline and post-intervention.

Results: The SIM group, but not the SEQ group, had a significant improvement on composite memory following the intervention $(\mathrm{t}(51)=2.7, p=0.01$, effect size $(\mathrm{ES})=0.42)$ and transfer to non-trained reasoning abilities $(\mathrm{t}(51)=6.0, \mathrm{ES}=0.49)$ and complex attention $(\mathrm{t}(51)=3.1, p=0.003$, ES $=0.70)$. Conversely, the SEQ group, but not the SIM, showed significant improvement in executive functioning $(\mathrm{t}(51)=5.0, p=0.0001, \mathrm{ES}=0.96)$.

Conclusion: These findings indicate that a 4-week simultaneous memory training and aerobic exercise program is sufficient to improve memory, attention, and reasoning abilities in older adults.

Keywords: Aerobic exercise, Alzheimer's disease, cognitive decline, cognitive training, dementia, memory training, physical activity, subjective memory complaints
\end{abstract}

\footnotetext{
${ }^{1}$ These authors contributed equally to this work.

*Correspondence to: Sarah C. McEwen, PhD, UCSD Department of Psychiatry, 9500 Gilman Drive, La Jolla, CA 92093, USA. Tel.: +1 858534 5927; E-mail: smcewen@ucsd.edu.
}

\section{INTRODUCTION}

According to the World Health Organization, 47.5 million people worldwide have dementia with an annual incidence of 7.7 million new cases every year. 
This number is projected to increase to 75.6 million people by 2030 and to 135.5 million people by 2050 [1]. With age being the single biggest non-modifiable risk factor for dementia and the lack of effective pharmacological treatment, several modifiable lifestyle factors have been identified and shown their potential beneficial effects in slowing cognitive decline [2]. Two factors that may affect cognitive performance and slow the progression of memory loss into dementia in older adults are cognitive training and physical activity $[2,3]$. Since treatment of dementia is still short of a cure, preventative strategies are of critical importance.

The effects of regular physical exercise, specifically aerobic exercise, have been associated with profound improvements in neurocognitive functioning in older adults and in neurodegenerative diseases $[4,5]$. Neurocognitive outcomes for exercise programs have generally been positive, as long as the exercise intervention was of adequate intensity and duration. However, a recent meta-analysis has revealed that intervention studies of single exercise interventions are discouraging and lacking in evidence to support the effects on cognition [6].

Enriched environments and more specifically cognitive training have also shown been shown to enhance cognitive performance and improve brain health in older adults $[7,8]$. More specifically in this population, strategy-based memory techniques were associated with improvements in memory performance whether used alone [9] or as part of a healthy lifestyle programs [10]. To date, few studies have assessed the effects of combining cognitive training and physical activity and results have demonstrated larger benefits on cognitive test performance for combined physical and cognitive activity than for each activity alone [11]. It has been proposed that exercise has to occur in the context of a cognitively challenged environment to be effective for inducing neural and cognitive benefits rather than exercise alone [12]. Furthermore, a recent meta-analysis that included eight studies showed that combined cognitive and exercise training can be effective for improving the cognitive functions and functional status of older adults with and without cognitive impairment [13]. Further, a combination of exercise and cognitive interventions, either sequentially or simultaneously, appears to have the potential effect to maintain or improve cognitive functions [14], but it is not known which is more effective at driving the changes. Therefore, more research is needed to understand the effects of simultaneous memory strategy training program during aerobic exercise. To our knowledge, only two recent studies have examined the synergistic effects of a combined intervention on cognitive performance in older adults $[15,16]$. Both studies have observed that the simultaneous training was associated with improved cognitive performance. However, one study did not have an active control group [15] and neither study used a strategy-based training approach for their simultaneous training, instead they used a drill-and-practice based approach, which is known not to improve transfer to cognitive skills or functional status [17]. In a study of healthy, young adults concurrently performing physical activity (aerobic cycling) during vocabulary word learning, they found that after nine training sessions ( 3 weeks) the simultaneous group, compared to a sedentary, non-exercise group, had better performance on the vocabulary test [18]. They also found electrophysiological recordings from the N400 component of ERPs, considered a signature of semantic processing, was increased post-intervention for the simultaneous training group. The authors interpret these data as showing that exercise improves encoding and retention for new vocabulary items by accelerating the learning process during exercise, which may be mediated by the N400 response. One recent study, which also examined the impact of simultaneous training versus sedentary training of a second language in young adults, found that the simultaneously training group had a more deeper understanding of the vocabulary words at the sentence level and also maintained their improved performance 1-month post-intervention [19]. Although these studies give initial evidence of the feasibility and efficacy of simultaneously learning while performing aerobic cycling, they are limited to studies of secondlanguage vocabulary word learning and also did not specifically target older adults, nor those with subjectively reported neurocognitive concerns.

The aim of this pilot study was to determine the temporal ordering of a short-term (i.e., 4-weeks/8 sessions) combined memory training and exercise intervention to determine if it is more effective to simultaneously combine these neuroprotective training strategies. In the present pilot study, we were interested in examining the primary outcome measure of memory functioning and also secondary outcomes of non-trained cognitive abilities of a novel strategy-based memory training program combined with an aerobic exercise program in older adults subjective memory impairments. We hypothesized that simultaneously combining these two treatments may 
make better use of brain-based plasticity mechanisms to rehabilitate cognitive deficits markedly better than separately undergoing either treatment.

\section{METHODS}

\section{Setting and participants}

In this two-site randomized controlled trial (RCT) study, a total of 92 non-demented healthy older adult volunteers (age 60-75) with subjective memory complaints were screened and enrolled into the study. The University of California, Los Angeles (UCLA) site in Westwood, CA enrolled 41 subjects, and the UCLA Health affiliated Motion Picture Television Fund (MPTF) campus site in Woodland Hills, CA enrolled 51 subjects. These participants were recruited from a variety of sources including, but not limited to, referrals from co-investigators and colleagues, previously enrolled UCLA Longevity Center research subjects that had agreed to be contacted about other research, advertising in the community, and faculty lectures in the community. Potential volunteers for the study were then screened by telephone to determine eligibility after oral consent was conducted. The inclusion and exclusion criteria asked over the phone included, but was not limited to, functional independence, health conditions, and medications. Additional measures were also completed over the phone including the Physical Activity Readiness Questionnaire for 70+ year-old adults (PAR-Q-70+), the Katz ADL scale, and the Lawton iADL scale. Those who met eligibility criteria were asked to come to either UCLA or MPTF for an intake visit and subsequent sessions (based on volunteer preferences and planned study group dates/openings). The study protocol was approved through the UCLA Institutional Review Board and was conducted in accordance with the ethical standards of the Committee on Human Experimentation at the UCLA and all participants provided signed informed consent.

\section{Study design and outcome measures}

This study was an RCT, in which participants were randomized to one of two active treatment conditions for a 4-week memory training and exercise intervention. Following the phone screening, participants were invited for an in-person screening visit and informed about the study's procedures, potential risks and benefits, and asked to provide their signed permission to participate in the study. Informed con- sent and screening procedures took place in a private interview room and were conducted by a study or nurse coordinator. The questionnaires screened for major health problems, including conditions limiting mobility, and assessed for vascular health risks. It also included a psychosocial and medical history administered by one of the study or nurse coordinators, which was reviewed by a study MD. All participants needed to receive clearance to participate in a moderate aerobic exercise program from their primary physician before they were enrolled in the study. Other measures used to quantify degree of health-related and cognitive impairments (and cutoff scores for inclusion) were: 1) Montreal Cognitive Assessment (MOCA) $\geq 23,2$ ) Stroke Risk (FSRP) Questionnaire, 3) STOP-BANG Questionnaire for Sleep Apnea, 4) Patient Health Questionnaire - 9 (PHQ-9) < 10, 5) Memory Function Questionnaire (MEM-Q 24; to quantify subjective memory impairment). The results of the above screening forms were reviewed by study staff for suitability to enroll in the study prior to proceeding further in the protocol. Resting blood pressure and pulse were then measured and recorded (these values were averaged with the measurements taken at the next baseline assessment visit). Additionally, height and weight were measured to establish body mass index (BMI) to compare differences in physical health at baseline between the groups. We also screened all the participants on the ability to complete a cardiovascular physical fitness test (YMCA 3-minute step test) prior to beginning the exercise ramp-up phase. However, changes in cardiovascular fitness were not an outcome measure used in this study, due to the short duration of the intervention (6 weeks), whereas other exercise intervention studies in older adults have found that detectable changes in cardiovascular fitness $\left(\mathrm{VO}_{2 \max }\right)$ from an aerobic training intervention need a minimum duration of at least 16-20+ weeks $[20,21]$.

\section{Neurocognitive battery}

All procedures outlined below took place in a private interview or patient room at the UCLA or MPTF site. Trained members of the research staff set-up participants to complete a standardized, computerized CNS Vital Signs Memory Protocol test at baseline and at end of study to assess neurocognitive function. CNS Vital Signs is a well-established neurocognitive and behavioral assessment tool whose psychometric properties have been validated and, specifically, 
is sensitive to deficits in mild cognitive dysfunction [22]. Participants performed the following eight tasks displayed on a desktop computer: 1) Verbal memory (word list learning), 2) Visual memory (figure learning) recognition (adaptations of the Rey Auditory Verbal and Visual List Learning Tests), 3) Finger tapping (adapted from the Halstead-Reitan Battery), 4) Symbol digit coding (based on the Symbol Digit Modalities Test, itself a variant of the Wechsler digit symbol substitution test), 5) Stroop test (had three parts that generate simple and complex reaction times), 6) Shifting attention (measures the subject's ability to shift from one instruction set (i.e., rules) to another quickly and accurately), 7) Continuous performance test (measures of vigilance or sustained attention), and 8) Non-verbal reasoning test (measured how well a subject can perceive and understand the meaning of visual or abstract information and recognizing relationships between visual-abstract concepts). Domain scores measured the following: composite memory (generated from the sum of correct responses from verbal and visual memory tests), verbal memory (correct hits and correct passes, immediate and delayed), visual memory (correct hits and correct passes, immediate and delayed), psychomotor speed (generated by totaling right and left taps from the finger tapping and total correct responses on the symbol digit coding), reaction time (generated by averaging the two complex reaction time scores from the Stroop Test), complex attention (generated by adding the number of errors committed in the continuous performance, the shifting attention and the Stroop), cognitive flexibility (generated by taking the number of correct responses on the shifting attention and subtracting the number of errors on the shifting attention and the Stroop Test), processing speed, executive function, motor speed, and simple attention.

\section{Randomization and intervention groups}

The study coordinator at the UCLA or MPTF sites directed the randomization and ramp-up procedure. Prior to beginning the formal exercise program, subjects were randomized $(1: 1)$ to the two study arms and then participated in a three-week exercise "rampup" phase. Both the SIM and SEQ groups (as defined below) completed up to two weeks of exercise only "ramp-up" consisting of twice weekly 1-hour sessions practicing stationary bicycling starting at 50\% heart rate reserve (HRR), which was calculated from the Karvonen method, for as long as they could tol- erate cycling (up to 30 minutes). Heart rate was continuously monitored via FitBit Charge HR using a wrist-worn heart rate monitor. During each session participants increased time and intensity by $5 \%$ (or as determined by the certified personal trainer-SM), until they could maintain 30 minutes of cycling at $65 \%$ HRR. Subjects who were unable to successfully complete ramp-up goals started the memory class at whatever level of fitness they had achieved by the end of ramp-up, and continued to work toward the goal of increasing from sustaining 50\% HRR to $65 \%$ HRR. Subjects who could not tolerate the ramp up exercise sessions were withdrawn from the study.

For the intervention, both the SIM and SEQ groups had two 2-hour sessions per week for 4 weeks (Fig. 1). The SIM subjects completed stretching/toning during the first hour; the SEQ completed aerobic cycling during the first hour, which included 10 minutes of warm-up (50\% HRR), 40 minutes of moderate intensity aerobic cycling (65\% HRR) and 10 minutes of cool-down $(<50 \%$ HRR). During the second half of the sessions, both groups first learned new memory training strategies while sedentary for 20 minutes. Then the SIM subjects then practiced the memory techniques for 40 minutes during stationary aerobic cycling at $65 \%$ HRR (Fig. 2) while SEQ subjects practiced the memory techniques while still sitting sedentary. Both groups then had group specific exercise and memory training homework assignments, along with monitoring of activity and logging of cognitively enriching activities. A trained study coordinator at the UCLA or MPTF site supervised the physical exercises and memory training sessions. The exercise sequence in relation to memory training and/or type of exercise is what varied between groups. The memory training course [10] combined presentations, memory checks, and skill-building exercises. Memory training was done either sitting sedentary or during stationary cycling. The memory training content was identical between the groups. Course material focused on addressing the following common memory complaints: forgetting names and faces, forgetting to do things in the future (e.g., remembering appointments or why you walked into a room), forgetting where you put things (e.g., keys, glasses, or a wallet), and inability to immediately recall something you know or "tip of the tongue" memory challenge. Subjects reviewed prior homework at check-in and were given new memory and stretching/toning exercise homework assignments at the end of each training session. 


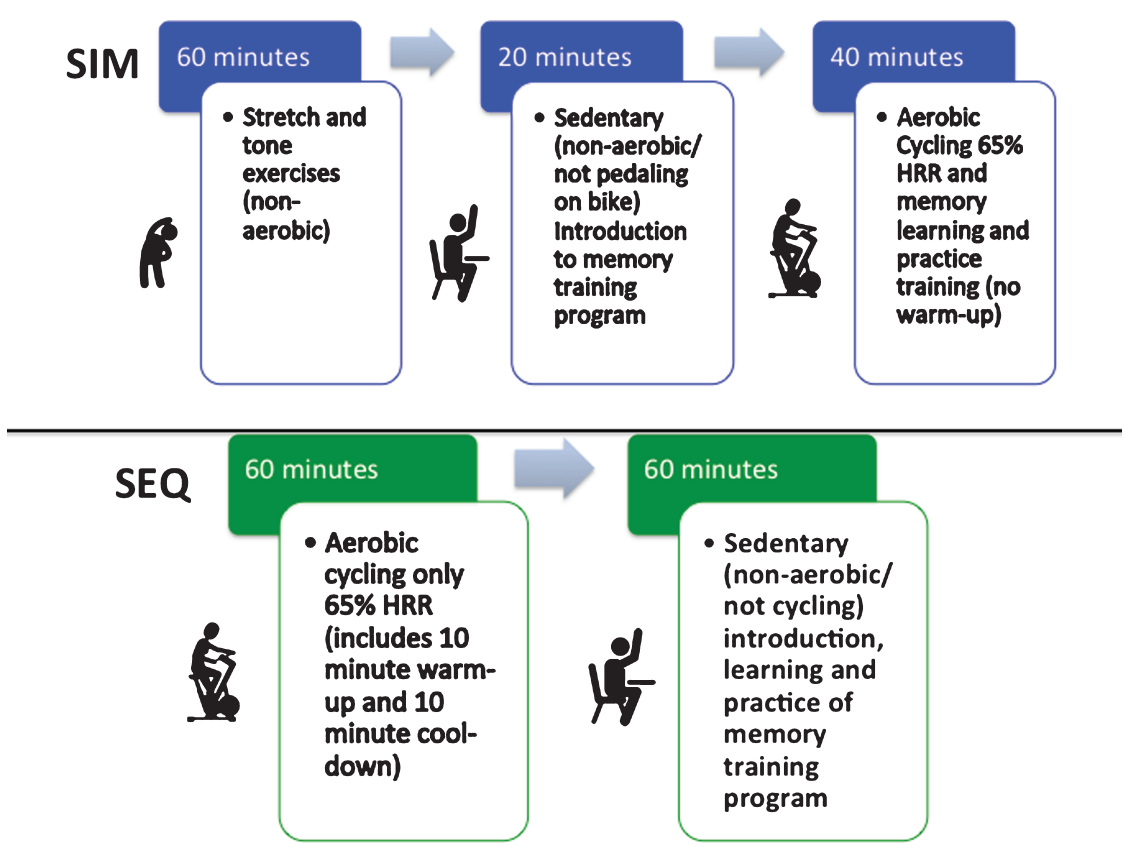

Fig. 1. Composition of the small group, trainer led, simultaneous (SIM) exercise and memory training or sequential (SEQ) exercise then memory training, 2-hour training sessions.

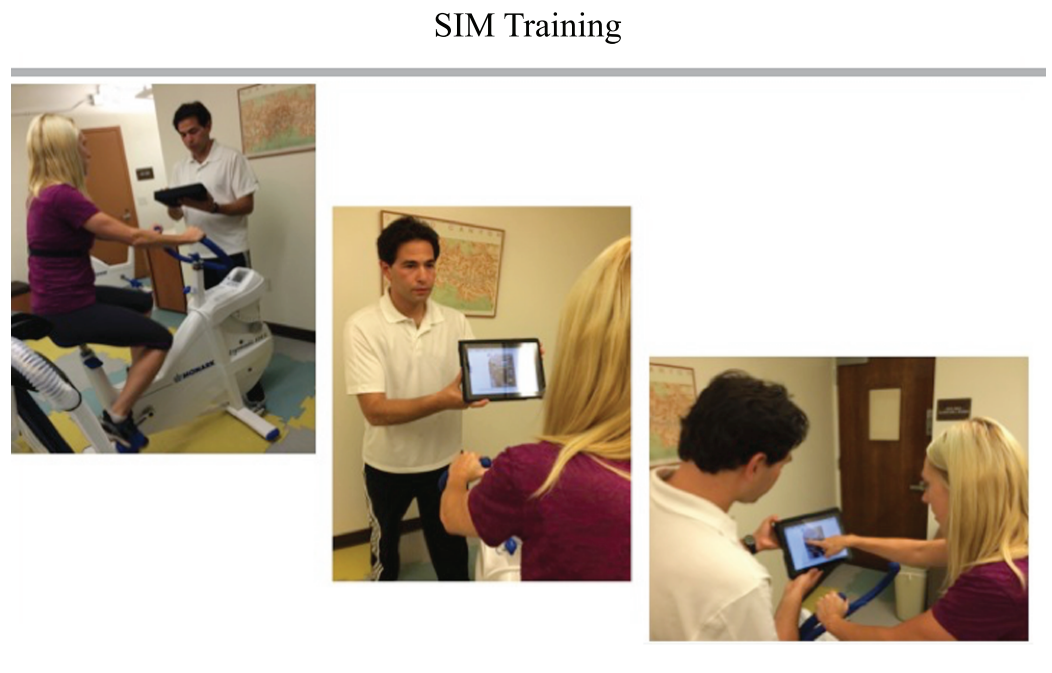

Fig. 2. Simultaneous (SIM) exercise and memory training session set-up. After completing 1 hour of stretch and tone (non-aerobic warm-up) movements to control for participant time in the sequential (SEQ) arm, SIM participants would complete their combined exercise session where they would have a 20-minute introduction to the memory training session for the day on the bike but not cycling (non-aerobic/sedentary). Then they would start pedaling on the stationary bike for 40 minutes of stationary, aerobic cycling (@ 65\% HRR) while learning the memory strategies and practicing the memory drills which were presented by the trainer on the screen in front of the class, this was followed by 5 minutes of cool-down on the bike.

\section{Statistical analysis}

All data were entered into the database at the time of their collection and analyzed after completion of the trial. Participants in the two arms were compared using $t$-tests (for continuous variables) or chi-squared tests (for categorical variables) on all demographic and clinical measures at baseline to assess the success of the randomization procedure. Site differences were compared using $t$-tests 
or chi-squared tests. All the outcome measures of interest are continuous and were analyzed using a mixed effects general linear model, as implemented in SAS PROC MIXED. The model included treatment arm as the between-subject factor, time as the withinsubject factor, and the interaction term between time and treatment arm as a predictor. Age, sex, and education were used as covariates and site was used as fixed effect. Post hoc analyses determined the significance of both between-group and within-group changes. Effect sizes (ES; Cohen's d) for changes in all outcome measures were estimated. The level of significance for outcomes was set at the alpha level of $p<0.05$, two-tailed. We did not employ any correction procedures for multiple comparisons, since this is the first study comparing SIM and SEQ to examine these measures; we are reporting the results of all analyses and our conclusions are based on the pattern of results. This approach thus permits the discovery of important features of the study findings without the reduction of power that would result from correction.

\section{RESULTS}

\section{Demographics and feasibility}

The CONSORT diagram for the study is shown in Fig. 3. After screening 126 subjects, 92 individuals met criteria for in-person screening and 76 were enrolled into the study and completed baseline testing, with 59 individuals beginning the study protocol. The protocol consisted of 1-2 weeks of observation, a ramping-up to exercise phase, and then the 4-week intervention. A total of 4 subjects dropped out ( 3 from SIM and 1 from SEQ) during the protocol. Drop-out rate did not differ by arm allocation. One SIM subject dropped out to an unrelated medical condition, another SIM and the one SEQ subject dropped out due to the time commitment, and the remaining SIM subject discontinued due to unknown reasons. No side effects or adverse events occurred. Mean adherence was $87.5 \%$ for the SIM group (Range: $50-100 \%$ ) and 93.3\% for the SEQ group (Range: $75-100 \%$ ) across the sites.

At completion, there were 29 participants in the SIM group and 26 in the SEQ group. The two intervention groups did not differ by age, gender, race, years of education, BMI, YMCA 3-minute step test (cardiovascular fitness; heart rate beats per minute), or cognitive status scores at baseline (Table 1). We also determined that participants in the two sites did not differ in in any of the demographic or clinical characteristics at baseline. We found that neither of the groups differed in baseline self-reported memory scores $(\mathrm{F}(1,51)=1.2, p=0.3)$, nor did they differ in changes in these scores $(\mathrm{F}(1,51)=0.03, p=0.9)$. Further, although both groups subjective memory scores improved from baseline to end of intervention neither of the groups showed significant changes, indicating the relative stability of this subjective measure that may not be sensitive to change over such a short intervention period.

\section{Cognitive outcomes}

There were no between-group differences in memory changes with time; however, SIM participants improved significantly on Composite Memory at the end of the intervention compared to baseline (mean change $=6.1(2.3), \mathrm{ES}=0.42, \mathrm{t}(51)=2.7$, $p=0.01)$ while there was no significant change in the SEQ group (mean change $=1.6(2.4), \mathrm{ES}=0.14$, $\mathrm{t}(51)=0.7, p=0.5$ ) (Fig. 4). Analyzing Verbal and Visual Memory separately, both the SIM (mean change $=8.4(2.6), \mathrm{ES}=0.48, \mathrm{t}(51)=3.2, p=0.003)$ and SEQ (mean change $=5.9$ (2.7), $\mathrm{ES}=0.46$, $\mathrm{t}(51)=2.2, \quad p=0.03)$ groups showed significantgroup improvements in Verbal Memory while neither group showed significant improvement in Visual Memory.

Changes in Executive Function abilities (performance on the shifting attention task) differed significantly between groups $(\mathrm{F}(1,51)=7.0$, $p=0.01$ ), favoring the SEQ group (mean change $=8.9$ (1.8), $\mathrm{ES}=0.96, \mathrm{t}(51)=5.0, p=0.0001)$ over the SIM Group (mean change $=2.4(1.7), \mathrm{ES}=0.31$, $\mathrm{t}(51)=1.4, p=0.2$ ) (Fig. 4). The two intervention groups differed significantly in their change in Reaction Time (performance speed on the Stroop test) $(\mathrm{F}(1,51)=4.6, p=0.04)$, with the SIM group showing no significant change (mean change $=-0.9(1.8)$, $\mathrm{ES}=-0.08, \mathrm{t}(51)=0.5, p=0.6$ ), while the SEQ group showed a significant increase (mean change $=4.7$ (1.9), $\mathrm{ES}=-0.08, \mathrm{t}(51)=2.5, p=0.01)$.

While no other changes in cognitive measures reached significance for the between -group comparisons, there were other significant within group changes, as shown in Table 2. Only the SIM group showed significant improvements in NonVerbal Abstract Reasoning (mean change $=6.0(2.5)$, $\mathrm{ES}=0.49, \mathrm{t}(51)=2.4, p=0.02)$ and Complex Attention (reduction in number of errors on the shifting attention, Stroop, and continuous performance tests) 


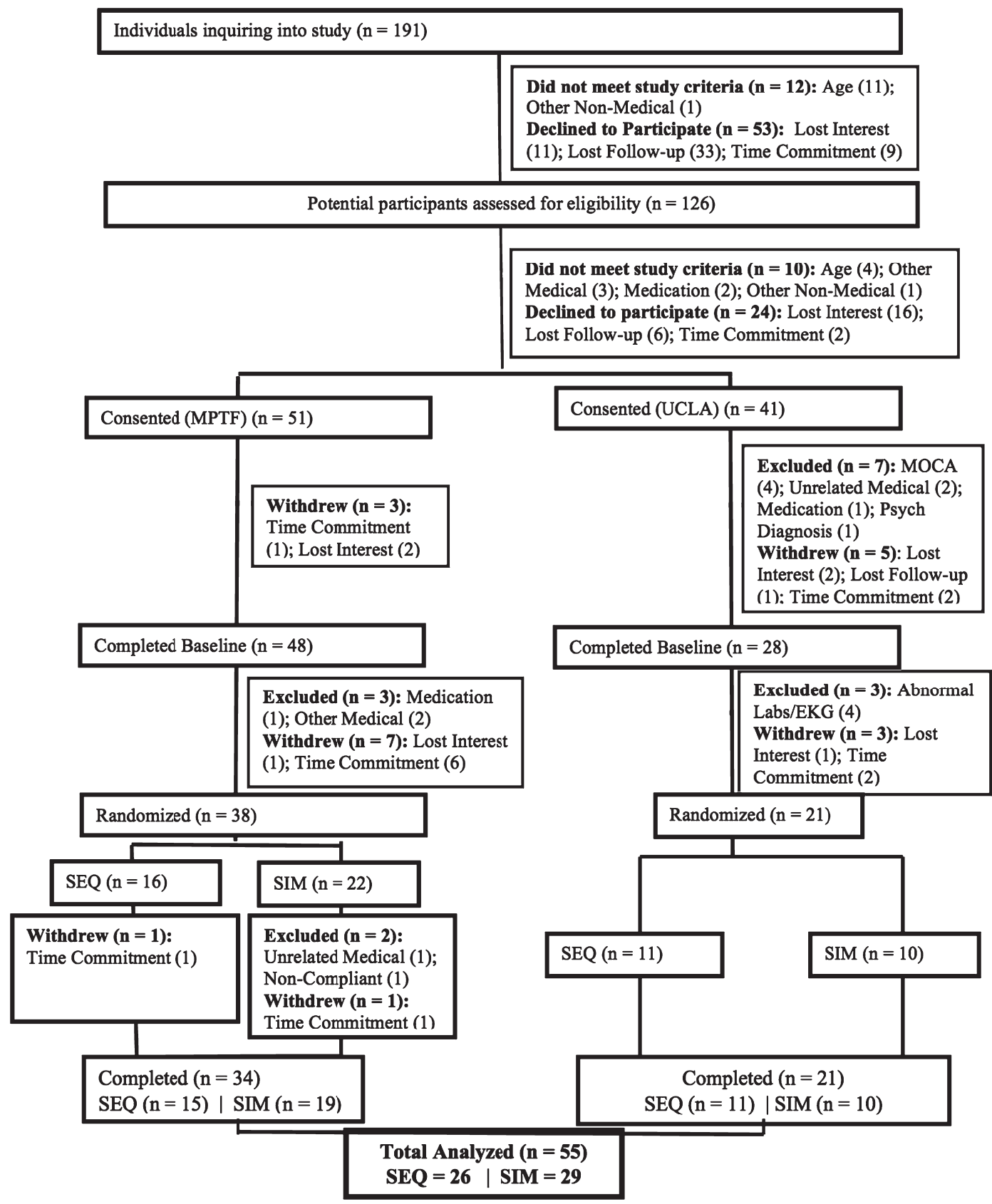

Fig. 3. CONSORT diagram and participant's flow.

(mean change $=9.7$ (3.1), $\mathrm{ES}=0.70, \mathrm{t}(51)=3.1$, $p=0.003$ ). Both SIM and SEQ showed significant improvement in Cognitive Flexibility (correct hits on the shifting attention and Stroop tests) (SIM: mean change $=4.7(2.0), \mathrm{ES}=0.53, \mathrm{t}(51)=2.4, p=0.02$; SEQ: mean change $=6.7(2.1), \mathrm{ES}=0.57, \mathrm{t}(51)=3.2$, $p=0.002$ ).

\section{DISCUSSION}

Our study is the first randomized intervention trial to examine the neurocognitive outcomes of either a simultaneous or sequentially administered strategy-based memory training program combined with an aerobic exercise program in older adults 
Table 1

Demographic characteristics* of sample

\begin{tabular}{|c|c|c|c|c|c|c|}
\hline & \multicolumn{2}{|c|}{ ALL } & \multicolumn{2}{|c|}{ UCLA } & \multicolumn{2}{|c|}{ MPTF } \\
\hline & SIM & SEQ & SIM & SEQ & SIM & SEQ \\
\hline$N$ & 29 & 26 & 10 & 11 & 19 & 15 \\
\hline Age & $67(5.1)$ & $65.4(3)$. & $66(4.5)$ & $64.7(3.0)$ & $67.5(5.4)$ & $65.9(4.5)$ \\
\hline Female $(\%)$ & $19(65.5)$ & $19(73.1)$ & $6(60)$ & $9(81.8)$ & $13(68.4)$ & $10(66.7)$ \\
\hline \multicolumn{7}{|l|}{ Race } \\
\hline Caucasian & $23(79.3)$ & $21(80.8)$ & $8(80.0)$ & $8(72.7)$ & $15(79.0)$ & $13(86.7)$ \\
\hline Black & $4(13.8)$ & $0(0)$ & $1(10)$ & $0(0)$ & $3(15.8)$ & $0(0)$ \\
\hline Asian American & $1(3.5)$ & $3(11.5)$ & $1(10)$ & $1(9.1)$ & $0(0)$ & $2(13.3)$ \\
\hline Other & $1(3.5)$ & $2(7.7)$ & $0(0)$ & $2(18.2)$ & $1(5.3)$ & $0(0)$ \\
\hline MOCA & $27.6(2.1)$ & $27.2(1.9)$ & $28.3(1.5)$ & $27.1(2.2)$ & $27.2(2.3)$ & $27.3(1.7)$ \\
\hline BMI & $27.1(5.4)$ & $25.6(3.9)$ & $25.0(5.0)$ & $26.6(3.9)$ & $28.3(5.3)$ & $24.9(3.9)$ \\
\hline MEM-Q 24 & $34.1(21.7)$ & $41.4(39.1)$ & $35.3(28.6)$ & $31.1(23)$ & $33.4(17)$ & $51.3(44.1)$ \\
\hline YMCA Step test & $101.5(18.3)$ & $101.1(13)$ & $108.9(21)$ & $112.4(13)$ & $93.8(15.5)$ & $89.8(12.7)$ \\
\hline
\end{tabular}

${ }^{*}$ Results are reported as mean (SD) for continuous variables and number of subjects (\%) for categorical variables. SIM, simultaneous exercise and memory training; SEQ, sequential exercise and memory training; MEM-Q 24, subjective memory questionnaire 24 items.

COMPOSITE MEMORY

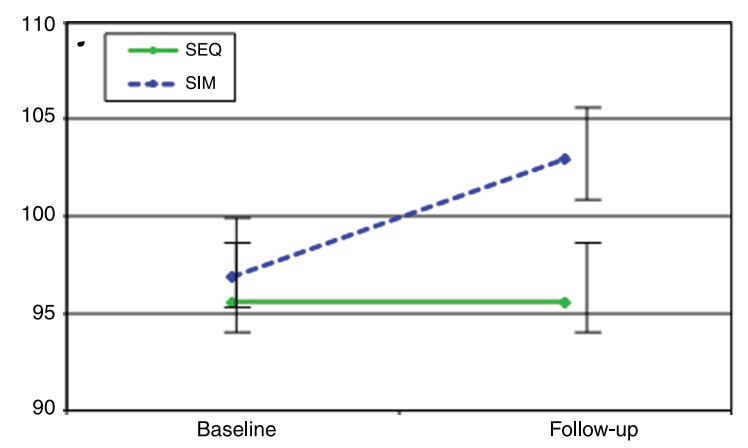

EXECUTIVE FUNCTIONING

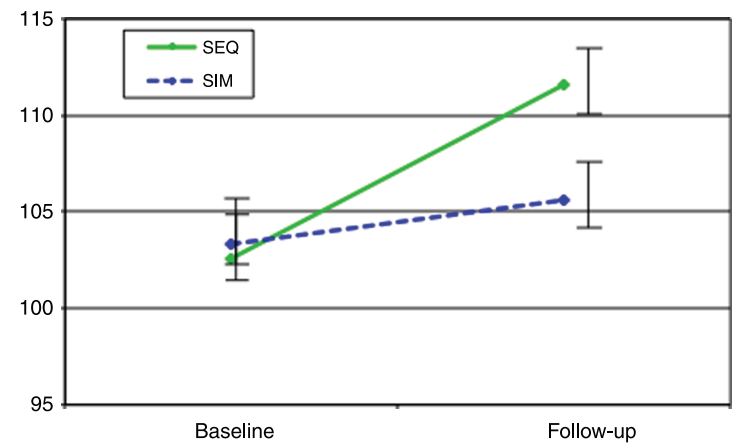

Fig. 4. Cognitive performance in Composite memory and Executive Functioning standard scores from baseline to immediately post-intervention for the simultaneous exercise and memory training (SIM) and sequential exercise and memory training (SEQ) groups. Error bars represent $95 \%$ confidence limits.

with subjective memory impairments. Our objectives were to show both the feasibility of the simultaneous training program and efficacy of this short intervention on improving memory functioning abilities to a greater extent than sequentially performing the training modalities. A large body of research in healthy and aged subjects demonstrates that regular physical exercise significantly improves general cognitive functioning, particularly verbal memory, executive functioning, processing speed, and visuospatial memory [23-25]. Another well-supported therapeutic approach to cognitive dysfunction is neuroplasticity-based cognitive training. Unfortunately, the generalizability of cognitive training to cognitive domains beyond those specifically targeted and its impact on real-world outcomes need to be strengthened [17]. Therefore, we developed a combined strategy-based memory and aerobic training program to determine the optimal temporal ordering of the cognitive training to impact our primary outcome of memory functioning and also secondary outcome measures of non-trained cognitive domains.

A goal of this pilot study was to show the feasibility and adoptability of the simultaneous exercise and memory training in older adults. We found that the drop-out rates were comparable between the SIM and SEQ groups, showing that the older adults in the SIM group were able to complete the memory training at the same level of engagement and ability as the SEQ group. Although we did not formally access satisfaction/enjoyment, by anecdotal report of the study trainers, participants of the SIM group more commonly reported that the time went faster during the SIM sessions which lead to more endorsement of enjoyment during those sessions.

Regarding our primary cognitive outcome of memory functioning changes, we found that 4 weeks of a memory training and aerobic exercise program was sufficient to improve composite/overall memory functioning in the group that did the training simultaneously. The composite memory tests assessed a range of memory abilities including how well indi- 
Table 2

Baseline and follow-up cognitive scores ${ }^{\#}$ of treatment groups

\begin{tabular}{|c|c|c|c|c|c|c|c|}
\hline \multirow[b]{2}{*}{ Domain } & \multicolumn{3}{|c|}{ SEQ } & \multicolumn{3}{|c|}{ SIM } & \multirow{2}{*}{$\begin{array}{l}\text { SIM versus } \\
\text { SEQ } \\
\text { ES }^{\#}\end{array}$} \\
\hline & $\begin{array}{c}\text { Baseline } \\
n=26\end{array}$ & $\begin{array}{c}\text { Follow-up } \\
n=26\end{array}$ & $\mathrm{ES}^{\$}$ & $\begin{array}{c}\text { Baseline } \\
n=29\end{array}$ & $\begin{array}{c}\text { Follow-up } \\
n=29\end{array}$ & $\mathrm{ES}^{\$}$ & \\
\hline Composite memory & $95.8(13.5)$ & $97.6(11.7)$ & 0.14 & $98.4(15.2)$ & $104.6(14.8)$ & $0.42^{*}$ & 0.45 \\
\hline Verbal memory & $96.3(13.3)$ & $102.3(13.2)$ & $0.46^{*}$ & $98.6(17.1)$ & $106.9(17.6)$ & $0.48^{*}$ & 0.15 \\
\hline Visual memory & $97.2(13.6)$ & $95.9(12.0)$ & -0.10 & $98.9(15.9)$ & $101.1(16.4)$ & 0.14 & 0.17 \\
\hline Psychomotor speed & $105.3(12.5)$ & $108.2(11.7)$ & 0.25 & $103.0(14.8)$ & $104.4(10.7)$ & 0.11 & -0.28 \\
\hline Reaction time & $96.8(14.1)$ & $101.5(12.3)$ & $0.35^{*}$ & $100.8(11.6)$ & $99.9(11.9)$ & -0.08 & $-0.54^{*}$ \\
\hline Complex attention & $102.5(10.2)$ & $106.9(16.0)$ & 0.33 & $96.5(17.9)$ & $106.3(8.2)$ & $0.70^{*}$ & 0.16 \\
\hline Cognitive flexibility & $101.3(10.1)$ & $108.2(13.5)$ & $0.57^{*}$ & $100.6(10.6)$ & $105.7(8.8)$ & $0.53^{*}$ & -0.14 \\
\hline Processing speed & $108.1(14.2)$ & $110.7(12.0)$ & 0.20 & $107.6(12.2)$ & $109.2(13.5)$ & 0.12 & -0.17 \\
\hline Executive functioning & $101.0(10.0)$ & $110.1(9.0)$ & $0.96^{*}$ & $103.0(9.2)$ & $105.9(9.0)$ & 0.31 & $-0.60^{*}$ \\
\hline Simple attention & $102.7(7.5)$ & $104.0(7.1)$ & 0.18 & $103.8(9.6)$ & $105.0(6.5)$ & 0.16 & -0.02 \\
\hline Motor speed & $101.7(12.2)$ & $104.0(14.1)$ & 0.18 & $99.2(16.0)$ & $100.0(11.8)$ & 0.06 & -0.27 \\
\hline Reasoning & $105.2(11.1)$ & $105.2(8.6)$ & 0.01 & $101.4(11.5)$ & $106.6(10.1)$ & $0.49^{*}$ & 0.54 \\
\hline
\end{tabular}

${ }^{\#}$ Results are reported as mean (SD); ${ }^{\$}$ ES, Effect size, within-group Cohen's d; ${ }^{\#}$ ES, Effect size, between-group Cohen's d; *Significant $(p$-value $<0.05)$.

viduals can recognize, remember, and retrieve words and geometric figures. These memory abilities are highly relevant and sensitive to decline in older adults' daily lives, being used to help remember appointments, taking medications, and the location of objects. These memory skills were the main focus of the strategy-based memory training program. Our findings are in-line with two recent studies that examined the synergistic effects of a simultaneous exercise and cognitive training intervention on cognitive performance in older adults $[16,26]$. Both studies observed that the simultaneous training was associated with improved cognitive performance. However, neither study used the more practical memory strategy-based training approach, and instead opted for the more commonly used drill-and-practice of basic neurocognitive skills-based approach. The memory training program used in this study was heavily concentrated on improving verbal memory learning, which is a skills known to significantly decline during aging [10]. We feel that there is a fundamental advantage of supplying participants with the skills needed to apply to memory strategies in real-life situations through the strategy-based training approaches. We therefore designed the study using a strategy-based training approach, in addition to also providing neuroplasticity-based drill-andpractice learning opportunities during each session. Such an approach may be more easily translated into their daily lives post-intervention.

Regarding the effects of the intervention on nontrained transfer of cognitive abilities we found that the SIM group, but not the SEQ group, showed significant improvements on the non-targeted skills:
Non-verbal reasoning and complex attention abilities. The skills assessed from the neurocognitive battery for the non-verbal reasoning test are thought to evaluate problem-solving abilities, discern meaning and insight and perceive relationships using abstract stimuli [22]. These higher order cognitive processes are a critical process of prefrontal cortex-mediated executive control network [27]. Research into the modulation of executive functioning performance during acute, low-to-moderate intensity exercise shows that performance is not impaired during the exercise training [28]; however during high-intensity exercise performance is significantly impaired [29]. These findings are in line with the transient hypofrontality theory during intense exercise [30]. Further, it has been suggested that within the context of the theoretical framework for plasticity, that acute physical activation may actually increase the range of flexibility for tasks that exceed the individual's capacity limits, especially impactful for those with lower cognitive functioning [15].

Although we did not explicitly target executive functioning or response time skills, as our main neurocognitive target was memory functioning in our cognitive training program, we found that the SEQ group improved significantly more than the SIM group in the composite executive functioning score and reaction time scores. These preferential findings for the SEQ training on these skills highlights that the transfer of these abilities from interventions of exercise and cognitive training which may best be targeted with separate training sessions, perhaps allowing for more allocation of mental resources and 
frontal engagement for participating in the task at hand. Since during the SIM training needing to perform memory training while cycling is an example of the executive functioning task of dual-tasking we may have overloaded attentional resources available, which could be maladaptive for the transfer of these skills. However, a study of dual-tasking abilities of visual-cognitive performance during acute exercise with treadmill walking at a preferred speed in older adults, actually showed better performance during the exercise than before or after [31]. This provides the rationale that steady-state moderate aerobic exercise may actually enhance executive functioning performance. Since our findings are not in line with others and we did have differences in baseline reaction time skills (significantly higher at baseline in the SIM group) and small group sizes it raises the need for future studies in larger, well-matched on cognition samples, to explore the transfer effects of the SIM training on executive functioning.

\section{Putative neurobiological mechanisms of SIM training}

We believe that simultaneous physical exercise and cognitive training may promote greater impact on neuroplasticity based on animal research which has found that combined aerobic exercise and cognitive enrichment yielded $\sim 30 \%$ greater increase in new neurons in rodents than either intervention alone [12]. They proposed that aerobic exercise "primes" increased neurogenesis in the dentate gyrus of the hippocampus when an animal is exposed to cognitive stimulation. Further, animal research suggests that exercise and cognitive training provide qualitatively different gains in neuroplasticity. Exercise supports proliferation and division of neuronal precursor cells in the dentate gyrus (expansion phase), whereas cognitive training promotes survival of these cells, implying an interdependence of the two treatments [32, 33]. Indeed, net neurogenesis is mostly determined not by the expansion phase but by cell survival [34]. In other words, new neurons created during physical exercise quickly die off when adequate learning opportunities or novel experiences do not accompany the increased physical activity [35-37]. Synaptic neuropil growth also depends on learning or novel experience, while increases in synaptic density and neuropil volume are relatively persistent $[37,38]$. For newly grown neurons to survive in the hippocampus, effortful learning must occur during cognitive training [39]. Therefore, our find- ing of greater memory improvement may be due to more effortful leaning that is occurring when the participants need to dual-task during the SIM session leading to greater neuroplastic benefits. Future trials need to be conducted to explore the neurobiological effects of SIM training on peripheral and in vivo biomarkers mediating the changes observed in cognitive functioning.

\section{Strengths and limitations}

This study has a number of strengths, including the use of randomization to assign participants to groups, that are sufficiently representative, sized and matched for a pilot study. We also had very high adherence rates providing the feasibility of this type of novel training in older adults. Lastly, we tested multiple cognitive abilities in addition to the centrally targeted memory performance skills. However, there are some limitations that need to be considered. One limitation of our study was that the physical training was monitored via heart rate monitor during training and we did not collect an objective gold-standard measure of cardiovascular fitness (i.e. $\mathrm{V0}_{2 \max }$ ) before and after the training. Therefore, we could not determine whether participant's aerobic capacity was increased as a result of the training. However, with the short duration of the intervention we would not have hypothesized that significant anthropomorphic or cardiovascular changes would be observed, nor was it the intention of the training (intensity was set at $65 \%$ maximal heart rate for the duration of the study) [20, 21]. Further, recent exercise intervention studies in older adults have not found a definitive, causal relationship between increases in fitness and cognitive improvements [40]. At least with respect to synergistic effects of simultaneous aerobic and memory training, increase of cardiovascular fitness does not necessarily represent a precondition. Additionally, the sample size of this study was limited due to the pilot nature of this project. Larger, longitudinal RCT studies of the effects of the simultaneous training are warranted in both non-clinical samples of older adults and those diagnosed with mild cognitive impairment to see if the improvements in memory and executive function still hold and also if the gains are sustained after the intervention has ceased and if the training attenuates the onset of dementia. Future studies also need to examine the neurobiological effects (i.e., MRI and peripheral blood samples) of the simultaneous training on brain plasticity to understand the underlying mechanisms driving cognitive changes. 


\section{ACKNOWLEDGMENTS}

This research was supported by the Director's Guild of America/Motion Picture and Television Fund (G.S.), the NIH National Center for Advancing Translational Sciences (UL1TR000124) (S.M.), NIMH Mentored Research Scientist Career Development Award K01 (MH099431) (S.M.) and the McLoughlin Gift Fund for Cognitive Health (D.M.). This RCT study is registered with NIH at clinicaltrials.gov (NCT02433691).

Authors' disclosures available online (https:// www.j-alz.com/manuscript-disclosures/17-0846r1).

\section{REFERENCES}

[1] World Health Organization. Dementia. Fact Sheets 2016; http://www.who.int/mediacentre/factsheets/fs362/en/. Accessed July 3, 2017.

[2] Merrill DA, Small GW (2011) Prevention in psychiatry: Effects of healthy lifestyle on cognition. Psychiatr Clin North Am 34, 249-261.

[3] Merrill DA, Siddarth P, Raji CA, Emerson ND, Rueda F, Ercoli LM, Miller KJ, Lavretsky H, Harris LM, Burggren AC, Bookheimer SY, Barrio JR, Small GW (2016) Modifiable risk factors and brain positron emission tomography measures of amyloid and tau in nondemented adults with memory complaints. Am J Geriatr Psychiatry 24, 729-737.

[4] Hotting K, Roder B (2013) Beneficial effects of physical exercise on neuroplasticity and cognition. Neurosci Biobehav Rev 37(9 Pt B), 2243-2257.

[5] Erickson KI, Voss MW, Prakash RS, Basak C, Szabo A, Chaddock L, Kim JS, Heo S, Alves H, White SM, Wojcicki TR, Mailey E, Vieira VJ, Martin SA, Pence BD, Woods JA, McAuley E, Kramer AF (2011) Exercise training increases size of hippocampus and improves memory. Proc Natl Acad Sci U S A 108, 3017-3022.

[6] Snowden M, Steinman L, Mochan K, Grodstein F, Prohaska TR, Thurman DJ, Brown DR, Laditka JN, Soares J, Zweiback DJ, Little D, Anderson LA (2011) Effect of exercise on cognitive performance in community-dwelling older adults: Review of intervention trials and recommendations for public health practice and research. $J$ Am Geriatr Soc 59, 704-716.

[7] Reijnders J, van Heugten C, van Boxtel M (2013) Cognitive interventions in healthy older adults and people with mild cognitive impairment: A systematic review. Ageing Res Rev 12, 263-275.

[8] Kelly ME, Loughrey D, Lawlor BA, Robertson IH, Walsh C, Brennan S (2014) The impact of cognitive training and mental stimulation on cognitive and everyday functioning of healthy older adults: A systematic review and meta-analysis. Ageing Res Rev 15, 28-43.

[9] Miller KJ, Dye RV, Kim J, Jennings JL, O’Toole E, Wong J, Siddarth P (2013) Effect of a computerized brain exercise program on cognitive performance in older adults. $A m J$ Geriatr Psychiatry 21, 655-663.

[10] Miller KJ, Siddarth P, Gaines JM, Parrish JM, Ercoli LM, Marx K, Ronch J, Pilgram B, Burke K, Barczak N, Babcock B, Small GW (2012) The memory fitness program:
Cognitive effects of a healthy aging intervention. Am J Geriatr Psychiatry 20, 514-523.

[11] Bamidis PD, Vivas AB, Styliadis C, Frantzidis C, Klados M, Schlee W, Siountas A, Papageorgiou SG (2014) A review of physical and cognitive interventions in aging. Neurosci Biobehav Rev 44, 206-220.

[12] Fabel K, Wolf SA, Ehninger D, Babu H, Leal-Galicia P, Kempermann G (2009) Additive effects of physical exercise and environmental enrichment on adult hippocampal neurogenesis in mice. Front Neurosci 3, 50.

[13] Law LL, Barnett F, Yau MK, Gray MA (2014) Effects of combined cognitive and exercise interventions on cognition in older adults with and without cognitive impairment: A systematic review. Ageing Res Rev 15, 61-75.

[14] Langdon KD, Corbett D (2012) Improved working memory following novel combinations of physical and cognitive activity. Neurorehabil Neural Repair 26, 523-532.

[15] Theill N, Schumacher V, Adelsberger R, Martin M, Jäncke L (2013) Effects of simultaneously performed cognitive and physical training in older adults. BMC Neurosci 14, 103.

[16] Eggenberger P, Schumacher V, Angst M, Theill N, de Bruin ED (2015) Does multicomponent physical exercise with simultaneous cognitive training boost cognitive performance in older adults? A 6-month randomized controlled trial with a 1-year follow-up. Clin Interv Aging 10, 13351349.

[17] Owen AM, Hampshire A, Grahn JA, Stenton R, Dajani S, Burns AS, Howard RJ, Ballard CG. (2010) Putting brain training to the test. Nature $\mathbf{4 6 5}, 775-778$.

[18] Schmidt-Kassow M, Kulka A, Gunter TC, Rothermich K, Kotz SA (2010) Exercising during learning improves vocabulary acquisition: Behavioral and ERP evidence. Neurosci Lett 482, 40-44.

[19] Liu F, Sulpizio S, Kornpetpanee S, Job R (2017) It takes biking to learn: Physical activity improves learning a second language. PLoS One 12, e0177624.

[20] Nelson ME, Rejeski WJ, Blair SN, Duncan PW, Judge JO, King AC, Macera CA, Castaneda-Sceppa C (2007) Physical activity and public health in older adults: Recommendation from the American College of Sports Medicine and the American Heart Association. Circulation 116, 1094-1105.

[21] Chodzko-Zajko WJ, Proctor DN, Fiatarone Singh MA, Minson CT, Nigg CR, Salem GJ, Skinner JS (2009) American College of Sports Medicine position stand. Exercise and physical activity for older adults. Med Sci Sports Exerc 41, 1510-1530.

[22] Gualtieri CT, Johnson LG (2006) Reliability and validity of a computerized neurocognitive test battery, CNS Vital Signs. Arch Clin Neuropsychol 21, 623-643.

[23] Colcombe S, Kramer AF (2003) Fitness effects on the cognitive function of older adults a meta-analytic study. Psychol Sci 14, 125-130.

[24] Hillman CH, Erickson KI, Kramer AF (2008) Be smart, exercise your heart: Exercise effects on brain and cognition. Nat Rev Neurosci 9, 58-65.

[25] Smith PJ, Blumenthal JA, Hoffman BM, Cooper H, Strauman TA, Welsh-Bohmer K, Browndyke JN, Sherwood A (2010) Aerobic exercise and neurocognitive performance: A meta-analytic review of randomized controlled trials. Psychosom Med 72, 239-252.

[26] Theill N, Schumacher V, Adelsberger R, Martin M, Jancke L (2013) Effects of simultaneously performed cognitive and physical training in older adults. BMC Neurosci 14, 103. 
[27] Koechlin E, Summerfield C (2007) An information theoretical approach to prefrontal executive function. Trends Cogn Sci 11, 229-235.

[28] Lambourne K, Audiffren M, Tomporowski PD (2010) Effects of acute exercise on sensory and executive processing tasks. Med Sci Sports Exerc 42, 1396-1402.

[29] Wang CC, Chu $\mathrm{CH}$, Chu IH, Chan KH, Chang YK (2013) Executive function during acute exercise: The role of exercise intensity. J Sport Exerc Psychol 35, 358-367.

[30] Dietrich A (2006) Transient hypofrontality as a mechanism for the psychological effects of exercise. Psychiatry Res 145, 79-83.

[31] Duncan MJ, Smith M, Clarke ND, Eyre EL, Wright SL (2016) Dual task performance in older adults: Examining visual discrimination performance whilst treadmill walking at preferred and non-preferred speeds. Behav Brain Res 302, 100-103.

[32] Kempermann G, Kuhn HG, Gage FH (1997) More hippocampal neurons in adult mice living in an enriched environment. Nature 386, 493-495.

[33] Kronenberg G, Reuter K, Steiner B, Brandt MD, Jessberger S, Yamaguchi M, Kempermann G. (2003) Subpopulations of proliferating cells of the adult hippocampus respond differently to physiologic neurogenic stimuli. J Comp Neurol 467, 455-463.
[34] Kempermann G, Chesler EJ, Lu L, Williams RW, Gage FH (2006) Natural variation and genetic covariance in adult hippocampal neurogenesis. Proc Natl Acad Sci U S A 103, 780-785.

[35] van Praag H, Christie BR, Sejnowski TJ, Gage FH (1999) Running enhances neurogenesis, learning, and long-term potentiation in mice. Proc Natl Acad Sci U S A 96, 1342713431.

[36] Kempermann G, Fabel K, Ehninger D, Babu H, Leal-Galicia P, Garthe A, Wolf SA (2010) Why and how physical activity promotes experience-induced brain plasticity. Front Neurosci 4, 189.

[37] Kleim JA, Markham JA, Vij K, Freese JL, Ballard DH, Greenough WT (2007) Motor learning induces astrocytic hypertrophy in the cerebellar cortex. Behav Brain Res 178, 244-249.

[38] Kleim JA, Swain RA, Czerlanis CM, Kelly JL, Pipitone MA, Greenough WT (1997) Learning-dependent dendritic hypertrophy of cerebellar stellate cells: Plasticity of local circuit neurons. Neurobiol Learn Mem 67, 29-33.

[39] Curlik DM, Shors TJ (2011) Learning increases the survival of newborn neurons provided that learning is difficult to achieve and successful. J Cogn Neurosci 23, 2159-2170.

[40] Thomas AG, Dennis A, Bandettini PA, Johansen-Berg H (2012) The effects of aerobic activity on brain structure. Front Psychol 3, 86. 